\title{
Xanthine stones
}

\section{Introduction}

Xanthine stones are absolutely rare. They are caused by an inborn defect of xanthine oxidase. Xanthine cannot be oxidized to uric acid, so that the excretion of hypoxanthine and xanthine increases. Xanthine is only poorly soluble, which results in xanthine stone formation.

A drug therapy to dissolve xanthine stones is not available. Consequently a high dilution day and night is the most important measure in xanthine stone disease. An ovo-lacto-vegetarian diet to increase the urinary $\mathrm{pH}$ value and to decrease xanthine excretion is also indicated. 


\section{Xanthine stones}
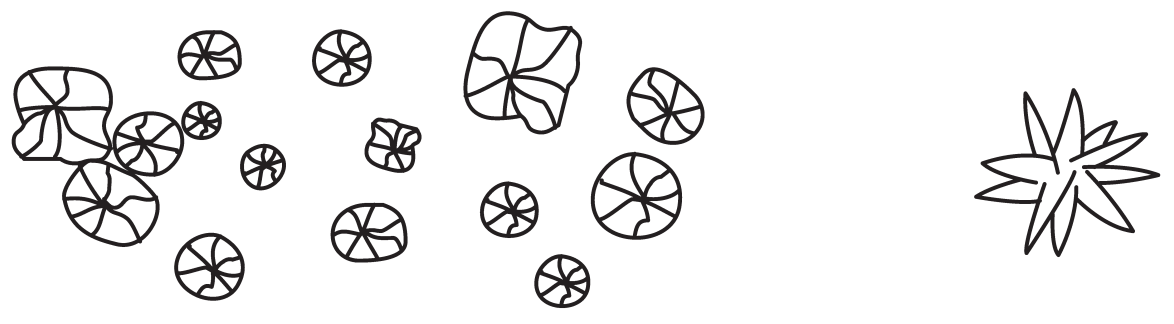

- Occurrence: very rare

- Caused by an autosomal recessive hereditary defect of the enzyme xanthine oxidase

- Lifelong consistent treatment is necessary

- Can easily be confused with uric acid stones 


\section{Specific notes for xanthine stone disease}

\section{Medical history}

Stones in family history

Treatment with high doses of allopurinol

Lesch-Nyhan disease

Chemotherapy of malignant diseases

Age at the first manifestation

Mostly in infancy

\section{Imaging}

Radiolucent

Contrast defect on the urogram

- Confusion with uric acid stones

Ultrasonography:

Structure with a high density and a typical echo

\section{Minimal program}

Serum analysis:

Urate markedly decreased $<119 \mu \mathrm{mol} / \mathrm{l}(2 \mathrm{mg} / 100 \mathrm{ml})$

\section{Establishment of the diagnosis only possible by}

stone analysis: Xanthine stone
$22 \mathrm{f}$.

24 f.

$26 \mathrm{f}$.

$192 \mathrm{f}$. 


\section{Quick reference with known serum and urine composition}

(in case of unclear findings, first episode of stone, etc.: consider the following pages on diagnostic work-up)

\section{Serum analysis}
Urate
$<119 \mu \mathrm{mol} / \mathrm{l}(<2 \mathrm{mg} / 100 \mathrm{ml})$

24h urine analysis

\begin{tabular}{|c|c|c|c|c|c|}
\hline Volume & $<2 \mathrm{l} / 24 \mathrm{~h}$ & D & (1) & Urine dilution & 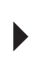 \\
\hline Urine density & $>1.010 \mathrm{~g} / \mathrm{cm}^{3}$ & 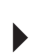 & I & Urine dilution & 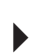 \\
\hline Xanthine & $>40 \mu \mathrm{mol} / 24 \mathrm{~h}$ & $\nabla$ & X & Diet & 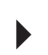 \\
\hline
\end{tabular}

Hypoxanthine $\quad>70 \mu \mathrm{mol} / 24 \mathrm{~h}$

กั

$\frac{0}{0}$

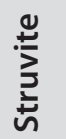

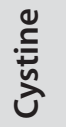

$\nwarrow$
$⿱$
0
0
0

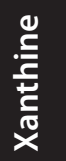

至旁

$\frac{x}{\frac{x}{0}}$
$\frac{0}{0}$
$\frac{0}{2}$ 


\section{Biochemical investigations}

\section{Serum analysis}

The striking and most important diagnostic finding is the markedly decreased serum level of urate. This is caused by the lack of activity of xanthine oxidase, which catalyzes the transformation of hypoxanthine to xanthine and xanthine to urate.

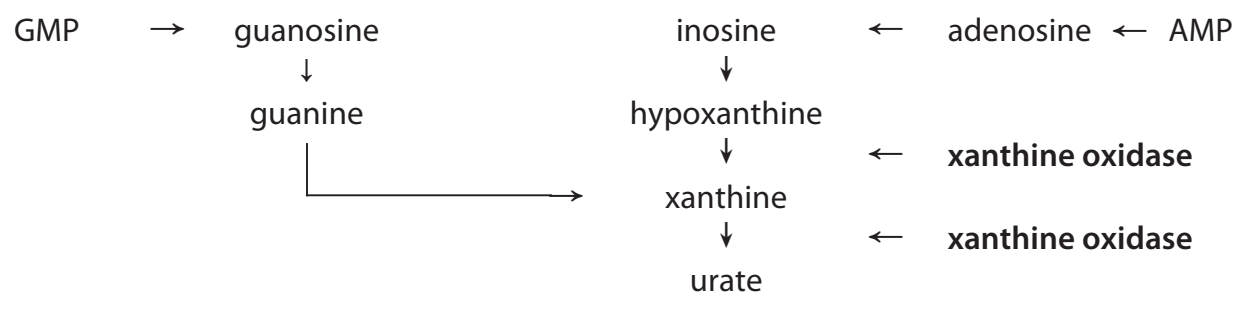

\section{Urine analysis}

\section{$24 \mathrm{~h}$ urine}

In addition to the analysis of the quality standard (see p. 28 f.), assessing the $24 \mathrm{~h}$ excretion of xanthine and hypoxanthine is essential for a correct diagnosis.

The patient has to collect a complete $24 \mathrm{~h}$ urine sample.

- Detailed instructions how to collect the sample are essential:

- the first portion of urine in the morning is discarded and the time noted

- collect all urine during the following $24 \mathrm{~h}$ in the bottle

- the last voiding should be made at the same time as the collection was started on the previous day

- if possible, store the urine in a cool place and bring it to analysis as soon as possible after the collection has been completed

(for detailed instructions how to collect urine, see p. 190)

Treatment with high doses of allopurinol in case of Lesch-Nyhan disease and with chemotherapy in malignant diseases can also lead to decreased serum levels of uric acid and an increased xanthine excretion with a risk of xanthine stone formation. To confirm the diagnosis of a primary xanthinuria, other medical reasons must be excluded. 
Urate markedly decreased $<119 \mu \mathrm{mol} / \mathrm{l}(<2 \mathrm{mg} / 100 \mathrm{ml})$

Normal value $119-380 \mu \mathrm{mol} / \mathrm{l}(2.0-6.4 \mathrm{mg} / 100 \mathrm{ml})$

\section{4h urine collection}

1. According to the quality standard

2. Quantitative determination of xanthine and hypoxanthine

$28 \mathrm{f}$.

196

very much increased

normal value $<40 \mu \mathrm{mol} / 24 \mathrm{~h}$

Caution: high doses of allopurinol may also cause xanthinuria

Hypoxanthine

normal or increased

normal value $<70 \mu \mathrm{mol} / 24 \mathrm{~h}$ 


\section{Treatment}

\section{Stone removal}

Chemolitholysis of xanthine stones is not possible. The symptoms caused by xanthine stones are less pronounced; therefore, at the time of diagnosis the stones often have reached a size which necessitates interventional measures. Because of the inborn error of metabolism with a risk of rapid stone growth, the aim should always be a complete surgical removal of all stones.

\section{Metaphylactic treatment}

At present, there are no possibilities for medical treatment of primary xanthinuria. Treatment with allopurinol is not successful. An increased urine dilution is particularly important.

\section{Urine dilution}

More than 2.5 I of urine should be excreted daily. The daily fluid intake should be 3 I for adults and $1.5-2$ I for children. Since extremely high concentrations of xanthine might occur during the sleeping period, the fluid intake should be evenly distributed over the $24 \mathrm{~h}$ period. It is a good habit to drink again before or after each voiding.

Therefore, in addition to the intake of other fluids:

- During day-time: drink at least $2.5 \mathrm{dl}$ every second hour

(approx. 2 cups or 2 small glasses)

During sleeping period: drink before going to bed and drink again before or after each voiding

\section{Diet}

Since xanthine originates from purine metabolism, a reduction of the dietary intake of purine is indicated. An ovolacto-vegetarian diet has a low purine content (see p. $82 \mathrm{f}$.) and is thus ideal for adults. In children, the requirements of protein, iron and iodine may not be sufficiently satisfied with such a diet. Thus, 1-2 meat meals and 1 fish meal should be given weekly. Foodstuff with extremely high purine content should always be avoided. 


\section{Stone removal}

- Xanthine stones are insoluble

- Indication for stone removal

$174 \mathrm{f}$.

- complaints and complications

- if metaphylactic therapy cannot prevent stone growth and new stone formation

Fluid intake: adults $3 \mathrm{l} /$ day, children $1.5-2 \mathrm{l} /$ day

evenly distributed over the $24 \mathrm{~h}$ period

\section{Adults: a diet with a low purine content}

- restrict: meat, meat products and fish (max. $150 \mathrm{~g} /$ day), legumes, including soy products

- avoid: innards, sardines, anchovies, sprats, mackerel, skin from fish, poultry and pork

\section{Children: do not completely exclude meat and fish from diet}

important for the supply of protein, iron, iodine 